\title{
Existence results for hybrid fractional neutral differential equations
}

\author{
Azmat Ullah Khan Niazi ${ }^{1 *}$, Jiang Wei ${ }^{1}$, Mujeeb Ur Rehman² and Du Jun ${ }^{1}$
}

\author{
"Correspondence: \\ azmatmath@yahoo.com \\ ${ }^{1}$ School of Mathematical Sciences, \\ Anhui University, Hefei, Anhui \\ 230039, China \\ Full list of author information is \\ available at the end of the article
}

\begin{abstract}
We discuss the existence of solutions of initial value problems for a class of hybrid fractional neutral differential equations. To prove the main results, we use a hybrid fixed point theorem for the sum of three operators. We also derive the dependence of a solution on the initial data and present an example to illustrate the results.
\end{abstract}

MSC: $34 \mathrm{~K} 37 ; 34 \mathrm{~B} 15$

Keywords: hybrid fractional neutral differential equations; initial value problem; hybrid fixed point theorem

\section{Introduction}

In the last two decades, many researchers attracted toward the study of fractional differential equations, motivated by their broad use in mathematical modeling. In the recent years, the theory of fractional differential equations has been analytically investigated by a large number of very interesting and novel papers; for some recent development on the topic, see [1-12] and the references therein.

Fractional calculus has found important applications in different fields, especially in problems related to acoustics, rheology or modeling of materials, thermal systems, and mechanical systems. Also, fractional differential equations have been used in models of biochemistry (modeling polymers and proteins), electrical engineering (transmission of ultrasound waves), medicine (modeling human tissue under mechanical loads), etc. Thus, differential and integral equations of fractional order play nowadays a very important role in describing some real-world phenomena [13-16].

Recently, quadratic perturbations of nonlinear fractional differential equations became a more interesting topic. Hybrid differential equations are important because they include several dynamic systems as particular cases. This class of hybrid fractional differential equations includes the perturbations of original differential equations in different ways. Much work has been done on the theory of hybrid differential equations; see the monographs [17-32]. Sitho et al. [27] discussed the existence of solutions by using the hybrid fixed point theorems of Dhage [24] for the sum of three operators in a Banach algebra for the following initial value problems of a hybrid fractional integro-differential equations:

$$
\left\{\begin{array}{l}
D_{0^{+}}^{\alpha}\left[\frac{x(t)-\sum_{i=1}^{m} I^{\beta} h_{i}(t, x(t))}{f(t, x(t))}\right]=g(t, x(t)), \quad t \in J=[0, T], \\
x(0)=0,
\end{array}\right.
$$

(c) The Author(s) 2017. This article is distributed under the terms of the Creative Commons Attribution 4.0 International License (http://creativecommons.org/licenses/by/4.0/), which permits unrestricted use, distribution, and reproduction in any medium, provided you give appropriate credit to the original author(s) and the source, provide a link to the Creative Commons license, and indicate if changes were made. 
where $D^{\alpha}$ denotes the Riemann-Liouville fractional derivative of order $\alpha, 0<\alpha \leq 1$, and $I^{\beta_{i}}$ is the Riemann-Liouville fractional integral of order $\beta_{i}>0$.

Zhao et al. [26] discussed the existence of solutions under mixed Lipschitz and Carathéodory conditions for the fractional hybrid differential equation

$$
\left\{\begin{array}{l}
D_{0^{+}}^{q}\left[\frac{x(t)}{f(t, x(t))}\right]=g(t, x(t)), \quad t \in J=[0, T], \\
x(0)=0,
\end{array}\right.
$$

where $D^{q}$ denotes the Riemann-Liouville fractional derivative of order $q$ with $0<q<1$, $f \in C(J \times \mathbb{R}, \mathbb{R} /\{0\})$, and $g \in C(J \times \mathbb{R}, \mathbb{R})$.

Moreover, the existence and uniqueness of a solution for the problem of type

$$
{ }^{c} D_{0^{+}}^{\gamma} x(t)=f\left(t, x_{t},{ }^{c} D_{0^{+}}^{\delta} x_{t}\right), \quad 0<t<1,
$$

where ${ }^{c} D_{0^{+}}^{\gamma}$ and ${ }^{c} D_{0^{+}}^{\delta}$ are Caputo derivatives with $1<\gamma<2$ and $0<\delta<1$, has already been proved by Niazi et al. [28] under some boundary conditions.

Motivated by these papers and the fact that the time-delay phenomenon is so common and certain. Many perturbations depend on both present and past status. Therefore, it is essential to consider the time-delay effect in the mathematical modeling of fractional differential equations. However, to the best of our knowledge, it looks like nobody considered the existence of a solution for the hybrid fractional neutral integro-differential equation (1). So, in this paper, we discuss the existence of a solution for the initial value problem for the hybrid fractional neutral integro-differential equation

$$
\begin{cases}{ }^{c} D_{0^{+}}^{\gamma}\left[\frac{x(t)-\sum_{i=1}^{m} I^{\zeta i} h_{i}\left(t, x_{t}\right)}{f\left(t, x_{t}\right)}\right]=g\left(t, x_{t},{ }^{c} D_{0^{+}}^{\delta} x_{t}\right), & t \in J=[0,1], \\ x(t)=\phi(t), & -\tau \leq t \leq 0,\end{cases}
$$

where ${ }^{c} D_{0^{+}}^{\gamma}$ and ${ }^{c} D_{0^{+}}^{\delta}$ are the Caputo derivatives with $0<\delta<\gamma<1, I^{\zeta_{i}}$ is the RiemannLiouville fractional integral of order $\zeta_{i}>0, i=1,2, \ldots, m, f \in C(J \times \mathbb{R}, \mathbb{R} /\{0\}), g \in C(J \times$ $\mathbb{R}, \mathbb{R})$, and $h_{i} \in C(J \times \mathbb{R}, \mathbb{R})$. Let $E=C(J, R)$ be the space of continuous real-valued functions defined on $J=[0,1]$. We define the norm $\|\cdot\|$ in $E$ for $0<\tau<1$ by $\|x\|=\sup \{|x(t)| ;-\tau \leq$ $t \leq 1\}$. For any $t \in[0,1]$, we define $x_{t}$ by $x_{t}(\theta)=x(t+\theta)$ for $\theta \in[-\tau, 0]$. As a motivation, problems of type (1) seem to be important in the study of dynamics of biological systems; see [33].

The paper is organized as follows. In Section 2, we recall some basic definitions. Section 3 is devoted to the existence of a solution for the IVP (1). Section 4 ends by proving the dependence of the solution on the initial value and its uniqueness. In Section 5, an example is given to explain the applicability of the results.

\section{Preliminaries}

This section contains some basic definitions and results.

Definition 2.1 ([13]) The gamma function is defined as

$$
\Gamma(\alpha)=\int_{0}^{\infty} e^{-t} t^{\alpha-1} d t, \quad \alpha>0
$$


One of the basic properties of the gamma function is the identity $\Gamma(\alpha+1)=\alpha \Gamma(\alpha)$.

Definition 2.2 ([13]) The Riemann-Liouville fractional integral of order $\alpha>0$ of a continuous function $f:(0, \infty) \rightarrow \mathbb{R}$ is defined by

$$
I^{\alpha} f(t)=\frac{1}{\Gamma(\alpha)} \int_{0}^{t}(t-s)^{\alpha-1} f(s) d s, \quad \alpha>0, t>t_{0}
$$

where $\Gamma$ is the gamma function, and right-hand side of the equality is defined pointwise on $\mathbb{R}^{+}$.

Definition 2.3 ([13]) The Caputo fractional derivative for a function $f:(0,+\infty) \rightarrow \mathbb{R}$ of order $\alpha(n-1<\alpha<n)$ is given by

$$
{ }^{c} D_{0^{+}}^{\alpha} f(t)=I_{0^{+}}^{n-\alpha} \frac{d^{n}}{d t^{n}} f(t)=\frac{1}{\Gamma(n-\alpha)} \int_{0^{+}}^{t} \frac{f^{(n)}(s)}{(t-s)^{\alpha+1-n}} d s, \quad t>0,
$$

where $n=[\alpha]+1([\alpha]$ stands for the bracket function of $\alpha)$.

Throughout the paper, $X$ denotes a Banach algebra with norm $\|\cdot\|$. The space $C(J, \mathbb{R})$ of all continuous functions endowed with the norm $\|x\|=\sup _{t \in J}|x(t)|$ is a Banach algebra. For proving the existence result, we will use the following fixed point theorem.

Lemma 2.1 [24] Let $S$ be a nonempty, closed convex, and bounded subset of a Banach algebra $X$, and let $P, R: X \rightarrow X$ and $Q: S \rightarrow X$ be three operators satisfying:

(i) $P$ and $R$ are Lipschitzian with Lipschitz constants $\delta$ and $\rho$, respectively,

(ii) $Q$ is compact and continuous,

(iii) $x=P x Q y+R x \Rightarrow x \in S$ for all $y \in S$,

(iv) $\delta M+\rho<1$, where $M=\|Q(S)\|$.

Then the operator equation $x=P x Q x+R x$ has a solution.

\section{Existence result}

We will use the following conditions:

(H1) $f$ is continuous, and for any $x, y \in \mathbb{R}$,

$$
\left|f\left(t, x_{t}\right)-f\left(t, y_{t}\right)\right| \leq l\left\|x_{t}-y_{t}\right\|, \quad \text { where } l \in[0,1]
$$

(H2) There exist a continuous function $\mu \in L^{\infty}\left(J, \mathbb{R}^{+}\right)$and a contraction $\psi: \mathbb{R}^{+} \rightarrow \mathbb{R}^{+}$ with contraction constant $<1$ and $\psi(0)=0$ such that, for $x \in R$ and $y \in \mathbb{R},|g(t, x, y)| \leq$ $\mu(t)(\|x\|+\|y\|)$ a.e., $t \in J$.

(H3) The functions $h_{i}: J \times \mathbb{R} \rightarrow \mathbb{R}, i=1,2, \ldots, m$, satisfy

$$
\left|h_{i}\left(t, x_{t}\right)-h_{i}\left(t, y_{t}\right)\right| \leq n_{i}\left\|x_{t}-y_{t}\right\|, \quad n_{i} \in[0,1]
$$

(H4) There exists $c>0$ such that, for $u_{i}, v_{i} \in \mathbb{R}, i=1,2$, and $s \in[0,1]$, we have

$$
\left|g\left(s, u_{1}, u_{2}\right)-g\left(s, v_{1}, v_{2}\right)\right| \leq c \sum_{i=1}^{2}\left|u_{i}-v_{i}\right|
$$


Lemma 3.1 Suppose that $0<\delta<\gamma<1$ and that functions $f, g, h_{i}, i=1,2, \ldots, m$, satisfy problem (1). Then the function $x \in R(J, \mathbb{R})$ is a solution of the hybrid fractional neutral integro-differential problem (1) if and only if it satisfies the integral equation

$$
\begin{aligned}
x(t)= & f\left(t, x_{t}\right)\left[\frac{\phi(0)-\sum_{i=1}^{m} I^{\zeta_{i}} h_{i}(0, \phi(\theta))}{f(0, \phi(\theta))}+\frac{1}{\Gamma(\gamma)} \int_{0}^{t}(t-s)^{\gamma-1} g\left(s, x_{s},{ }^{c} D_{0^{+}}^{\delta} x_{s}\right) d s\right] \\
& +\sum_{i=1}^{m} I^{\zeta_{i}} h_{i}\left(t, x_{t}\right) .
\end{aligned}
$$

Proof Applying $I^{\gamma}$ to both sides of equation (1), we get

$$
\frac{x(t)-\sum_{i=1}^{m} I^{\zeta i} h_{i}\left(t, x_{t}\right)}{f\left(t, x_{t}\right)}-\frac{x(0)-\sum_{i=1}^{m} I^{\zeta i} h_{i}\left(0, x_{0}\right)}{f\left(0, x_{0}\right)}=\frac{1}{\Gamma(\gamma)} \int_{0}^{t}(t-s)^{\gamma-1} g\left(s, x_{s},{ }^{c} D_{0^{+}}^{\delta} x_{s}\right) d s,
$$

and

$$
\begin{gathered}
\frac{x(t)-\sum_{i=1}^{m} I^{\zeta_{i}} h_{i}\left(t, x_{t}\right)}{f\left(t, x_{t}\right)}-\frac{\phi(0)-\sum_{i=1}^{m} I^{\zeta_{i}} h_{i}(0, \phi(\theta))}{f(0, \phi(\theta))} \\
=\frac{1}{\Gamma(\gamma)} \int_{0}^{t}(t-s)^{\gamma-1} g\left(s, x_{s},{ }^{c} D_{0^{+}}^{\delta} x_{s}\right) d s
\end{gathered}
$$

for $\theta \in[-\tau, 0]$. Hence

$$
\begin{aligned}
x(t)= & f\left(t, x_{t}\right)\left[\frac{\phi(0)-\sum_{i=1}^{m} I^{\zeta_{i}} h_{i}(0, \phi(\theta))}{f(0, \phi(\theta))}+\frac{1}{\Gamma(\gamma)} \int_{0}^{t}(t-s)^{\gamma-1} g\left(s, x_{s},{ }^{c} D_{0^{+}}^{\delta} x_{s}\right) d s\right] \\
& +\sum_{i=1}^{m} I^{\zeta_{i}} h_{i}\left(t, x_{t}\right) .
\end{aligned}
$$

Theorem 3.1 Assume that (H1)-(H3) hold. Assume that there exists a real number $v$ such that

$$
v \geq \frac{F_{0}\left[N+\frac{\mu(s)\left(\left(1+\frac{1}{\Gamma(2-\delta)}\right) v\right)}{\Gamma(\gamma+1)}\right]+\sum_{i=1}^{m} \frac{K_{0}}{\Gamma\left(\zeta_{i}+1\right)}}{1-N l-l \frac{\mu(s)\left(\left(1+\frac{1}{\Gamma(2-\delta)}\right) v\right)}{\Gamma(\gamma+1)}-\sum_{i=1}^{m} \frac{n_{i}}{\Gamma\left(\zeta_{i}+1\right)}},
$$

where $F_{0}=\sup _{t \in J}|f(t, 0)|$ and $K_{0}=\sup _{t \in J}\left|h_{i}(t, 0)\right|, i=1,2, \ldots, m, N=\left|\frac{\phi(0)-\sum_{i=1}^{m} I \zeta_{i} h_{i}(0, \phi(\theta))}{f(0, \phi(\theta))}\right|$, and

$$
N l+l \frac{\mu(s)\left(\left(1+\frac{1}{\Gamma(2-\delta)}\right) v\right)}{\Gamma(\gamma+1)}+\sum_{i=1}^{m} \frac{n_{i}}{\Gamma\left(\zeta_{i}+1\right)}<1 .
$$

Then problem (1) has at least one solution on J.

Proof Take $X=R(J, \mathbb{R})$ and define the subset $S$ of $X$ as

$$
S=\left\{x \in X:\left\|x_{t}\right\| \leq v\right\},
$$


where $v$ satisfies equation (3). Clearly, $S$ is a closed, convex, and bounded subset of the Banach space $X$. By Lemma 3.1, problem (1) is equivalent to the integral equation (2). Now we define three operators: $P: X \rightarrow X$ by

$$
P x(t)=f\left(t, x_{t}\right), \quad t \in J,
$$

$Q: S \rightarrow X$ by

$$
\begin{aligned}
Q x(t)= & \frac{\phi(0)-\sum_{i=1}^{m} I^{\zeta i} h_{i}(0, \phi(\theta))}{f(0, \phi(\theta))} \\
& +\frac{1}{\Gamma(\gamma)} \int_{0}^{t}(t-s)^{\gamma-1} g\left(s, x_{s},{ }^{c} D_{0^{+}}^{\delta} x_{s}\right) d s \quad \text { for } \theta \in[-\tau, 0],
\end{aligned}
$$

and $R: X \rightarrow X$ by

$$
R x(t)=\sum_{i=1}^{m} I^{\zeta_{i}} h_{i}\left(t, x_{t}\right), \quad t \in J
$$

We shall show that the operators $P, Q$, and $R$ satisfy all the conditions of Lemma 2.1. This will be achieved in the following series of steps.

Step 1. We will show that $P$ and $R$ are Lipschitzian on $X$. Let $x, y \in X$. Then, for $t \in J$, we have

$$
\begin{aligned}
|P x(t)-P y(t)| & =\left|f\left(t, x_{t}\right)-f\left(t, y_{t}\right)\right| \\
& \leq l\left\|x_{t}-y_{t}\right\| \leq l \sup _{0 \leq t \leq 1}|x(t+\theta)-y(t+\theta)|, \quad \theta \in[-\tau, 0] \\
& \leq l \sup _{-\tau \leq t+\theta \leq 1}|x(t+\theta)-y(t+\theta)| \\
& \leq l \sup _{-\tau \leq t^{\prime} \leq 1}\left|x\left(t^{\prime}\right)-y\left(t^{\prime}\right)\right|, \quad t^{\prime}=t+\theta \\
\Rightarrow\|P x-P y\| & =l\|x-y\| \quad \text { for all } x, y \in X .
\end{aligned}
$$

Therefore, $P$ is Lipschitzian on $X$ with Lipschitz constant $l$. Also, for any $x, y \in X$, we have

$$
\begin{aligned}
|R x(t)-R y(t)| & =\left|\sum_{i=1}^{m} I^{\zeta_{i}} h_{i}\left(t, x_{t}\right)-\sum_{i=1}^{m} I^{\zeta_{i}} h_{i}\left(t, y_{t}\right)\right| \\
& \leq \sum_{i=1}^{m} \int_{0}^{t} \frac{(t-s)^{\gamma-1}}{\Gamma\left(\zeta_{i}\right)}\left|h_{i}\left(s, x_{s}\right)-h_{i}\left(s, y_{s}\right)\right| d s \\
& \leq \sum_{i=1}^{m} \int_{0}^{t} \frac{(t-s)^{\gamma-1}}{\Gamma\left(\zeta_{i}\right)} n_{i}\left\|x_{s}-y_{s}\right\| d s \\
& \leq\|x-y\| \sum_{i=1}^{m} \frac{n_{i}}{\Gamma\left(\zeta_{i}\right)} \frac{t^{\zeta_{i}}}{\zeta_{i}} \\
\Rightarrow\|R x-R y\| & =\sum_{i=1}^{m} \frac{n_{i}}{\Gamma\left(\zeta_{i}+1\right)}\|x-y\| \quad \text { for all } x, y \in X .
\end{aligned}
$$


Thus, $R$ is a Lipschitzian on $X$ with Lipschitz constant $\sum_{i=1}^{m} \frac{n_{i}}{\Gamma\left(\zeta_{i}+1\right)}$.

Step 2. Now we will prove that the operator $Q$ is completely continuous on $X$. First, we show that $Q$ is continuous on $S$. Let $\left\{x_{n}\right\}$ be a sequence in $S$ converging to a point $x \in S$. Then by the Lebesgue dominated convergence theorem

$$
\begin{aligned}
\lim _{n \rightarrow \infty} Q x_{n}(t) & =\frac{\phi(0)-\sum_{i=1}^{m} I^{\zeta_{i}} h_{i}(0, \phi(\theta))}{f(0, \phi(\theta))}+\lim _{n \rightarrow \infty} \frac{1}{\Gamma(\gamma)} \int_{0}^{t}(t-s)^{\gamma-1} g\left(s, x_{n s},{ }^{c} D_{0^{+}}^{\delta} x_{n s}\right) d s \\
& =\frac{\phi(0)-\sum_{i=1}^{m} I^{\zeta_{i}} h_{i}(0, \phi(\theta))}{f(0, \phi(\theta))}+\frac{1}{\Gamma(\gamma)} \int_{0}^{t}(t-s)^{\gamma-1} \lim _{n \rightarrow \infty} g\left(s, x_{n s},{ }^{c} D_{0^{+}}^{\delta} x_{n s}\right) d s \\
& =\frac{\phi(0)-\sum_{i=1}^{m} I^{\zeta_{i}} h_{i}(0, \phi(\theta))}{f(0, \phi(\theta))}+\frac{1}{\Gamma(\gamma)} \int_{0}^{t}(t-s)^{\gamma-1} g\left(s, x_{s},{ }^{c} D_{0^{+}}^{\delta} x_{s}\right) d s \\
& =Q x(t)
\end{aligned}
$$

for all $t \in J$. Hence, $Q$ is a continuous operator on $S$. Now we will show that $Q$ is a compact operator on $S$. We have to prove that $Q(s)$ is a uniformly bounded and equicontinuous set in $X$. By $(\mathrm{H} 2)$ we have

$$
\begin{aligned}
|Q x(t)| & =\left|\frac{\phi(0)-\sum_{i=1}^{m} I^{\zeta_{i}} h_{i}(0, \phi(\theta))}{f(0, \phi(\theta))}\right|+\left|\frac{1}{\Gamma(\gamma)} \int_{0}^{t}(t-s)^{\gamma-1} g\left(s, x_{s},{ }^{c} D_{0^{+}}^{\delta} x_{s}\right) d s\right| \\
& \leq N+\frac{1}{\Gamma(\gamma)} \int_{0}^{t}(t-s)^{\gamma-1} \mu(s)\left(\left\|x_{s}\right\|+\left\|D_{0^{+}}^{\delta} x_{s}\right\|\right) d s \\
& \leq N+\frac{1}{\Gamma(\gamma)} \int_{0}^{t}(t-s)^{\gamma-1} \mu(s)\left(\left\|x_{s}\right\|+\frac{1}{\Gamma(2-\delta)}\left\|x_{s}\right\|\right) d s \\
& \leq N+\frac{1}{\Gamma(\gamma+1)} \mu(s)\left(1+\frac{1}{\Gamma(2-\delta)}\right) v=K_{1}
\end{aligned}
$$

for all $t \in J$. Therefore, $\|Q\| \leq K_{1}$, which shows that $Q$ is uniformly bounded on $S$. Now, we will show that $Q(s)$ is an equicontinuous set in $X$. Let $t_{1}, t_{2} \in J$ with $t_{1}<t_{2}$. Then, for any $x \in S$,

$$
\begin{aligned}
\left|Q x\left(t_{2}\right)-Q x\left(t_{1}\right)\right| \leq & \mid \frac{1}{\Gamma(\gamma)} \int_{0}^{t_{2}}\left(t_{2}-s\right)^{\gamma-1} g\left(s, x_{s},{ }^{c} D_{0^{+}}^{\delta} x_{s}\right) d s \\
& -\frac{1}{\Gamma(\gamma)} \int_{0}^{t_{1}}\left(t_{1}-s\right)^{\gamma-1} g\left(s, x_{s},{ }^{c} D_{0^{+}}^{\delta} x_{s}\right) d s \mid \\
\leq & \frac{1}{\Gamma(\gamma)} \int_{0}^{t_{1}}\left[\left(t_{2}-s\right)^{\gamma-1}-\left(t_{1}-s\right)^{\gamma-1}\right]\left|g\left(s, x_{s},{ }^{c} D_{0^{+}}^{\delta} x_{s}\right)\right| d s \\
& +\frac{1}{\Gamma(\gamma)} \int_{t_{1}}^{t_{2}}\left(t_{2}-s\right)^{\gamma-1}\left|g\left(s, x_{s},{ }^{c} D_{0^{+}}^{\delta} x_{s}\right)\right| d s \\
\leq & \frac{1}{\Gamma(\gamma+1)}\left[\left(t_{2}\right)^{\gamma}-\left(t_{1}\right)^{\gamma}-\left(t_{2}-t_{1}\right)^{\gamma}\right] \mu(s)\left(1+\frac{1}{\Gamma(2-\delta)}\right)(v) \\
& +\frac{\left(t_{2}-t_{1}\right)^{\gamma}}{\Gamma(\gamma+1)} \mu(s)\left(1+\frac{1}{\Gamma(2-\delta)}\right)(v) \\
\leq & \frac{1}{\Gamma(\gamma+1)}\left[\left(t_{2}\right)^{\gamma}-\left(t_{1}\right)^{\gamma}\right] \mu(s)\left(1+\frac{1}{\Gamma(2-\delta)}\right) v,
\end{aligned}
$$


which is independent of $x \in S$. As $t_{1} \rightarrow t_{2}$, the right-hand side of the last inequality tends to zero. Therefore, it follows from the Arzelà-Ascoli theorem that $Q$ is a completely continuous operator on $S$.

Step 3. We will prove that hypothesis (iii) of Lemma 2.1 is satisfied, that is, $x=P x Q y+$ $R x \Longrightarrow x \in S$ for all $y \in S$. We have

$$
\begin{aligned}
|x(t)| \leq & |P x(t)||Q y(t)|+|R x(t)| \\
\leq & \left|f\left(t, x_{t}\right)\right|\left\{\left|\frac{\phi(0)-\sum_{i=1}^{m} I^{\zeta_{i}} h_{i}(0, \phi(\theta))}{f(0, \phi(\theta))}\right|+\frac{1}{\Gamma(\gamma)}\left|\int_{0}^{t}(t-s)^{\gamma-1} g\left(s, y_{s}{ }^{c} D_{0^{+}}^{\delta} y_{s}\right) d s\right|\right\} \\
& +\left|\sum_{i=1}^{m} I^{\zeta_{i}} h_{i}\left(t, x_{t}\right)\right| \\
\leq & \left(\left|f\left(t, x_{t}\right)-f(t, 0)\right|+|f(t, 0)|\right)\left\{N+\frac{1}{\Gamma(\gamma+1)} \mu(s)\left(1+\frac{1}{\Gamma(2-\delta)}\right)(v)\right\} \\
& +\sum_{i=1}^{m} \int_{0}^{t} \frac{(t-s)^{\zeta_{i}-1}}{\Gamma\left(\zeta_{i}\right)} \\
& \times\left(\left|h_{i}\left(s, x_{s}\right)-h_{i}(s, 0)\right|+\left|h_{i}(s, 0)\right|\right) d s \\
\leq & \left(l\left\|x_{t}\right\|+F_{0}\right)\left\{N+\frac{1}{\Gamma(\gamma+1)} \mu(s)\left(1+\frac{1}{\Gamma(2-\delta)}\right)(v)\right\}+\sum_{i=1}^{m} \frac{n_{i}\left\|x_{t}\right\|+K_{0}}{\Gamma\left(\zeta_{i}+1\right)} \\
\leq & \left(l v+F_{0}\right)\left\{N+\frac{1}{\Gamma(\gamma+1)} \mu(s)\left(1+\frac{1}{\Gamma(2-\delta)}\right)(v)\right\}+\sum_{i=1}^{m} \frac{n_{i} v+K_{0}}{\Gamma\left(\zeta_{i}+1\right)} \leq v,
\end{aligned}
$$

which implies that $\|x\| \leq v$, and therefore $x \in S$.

Step 4. Finally, we will show that $v M+\rho<1$, that is, (iv) of Lemma 2.1 holds. Since

$$
\begin{aligned}
M & =\|Q(s)\|=\sup _{t \in J}\left|N+\frac{1}{\Gamma(\gamma)} \int_{0}^{t}(t-s)^{\gamma-1} g\left(s, x_{s},{ }^{c} D_{0^{+}}^{\delta} x_{s}\right) d s\right| \\
& \leq N+\frac{1}{\Gamma(\gamma+1)} \mu(s)\left(1+\frac{1}{\Gamma(2-\delta)}\right)(v),
\end{aligned}
$$

using equation (3), we have

$$
\begin{aligned}
\nu M+\rho & =l M+\sum_{i=1}^{m} \frac{n_{i}}{\Gamma\left(\zeta_{i}+1\right)} \\
& =l N+\frac{l \mu(s)\left(1+\frac{1}{\Gamma(2-\delta)}\right)(v)}{\Gamma(\gamma+1)}+\sum_{i=1}^{m} \frac{n_{i}}{\Gamma\left(\zeta_{i}+1\right)}<1
\end{aligned}
$$

with $v=l$ and $\rho=\sum_{i=1}^{m} \frac{n_{i}}{\Gamma\left(\zeta_{i}+1\right)}$. Thus all the conditions of Lemma 2.1 are satisfied, and hence the operator equation $x=P x Q x+R x$ has a solution in $S$. In consequence, problem (1) has a solution on $J$. This completes the proof.

\section{Data dependence of solution}

In this section, we will derive the data dependence of a solution for equation (1). 
Theorem 4.1 Let $x$ and $y$ be two solutions to the fractional hybrid equation (1) with $\phi=\phi_{1}$ and $\phi=\phi_{2}$, respectively. Then we have

$$
\begin{aligned}
\|x-y\| \leq & {\left[l\left[N+\frac{\mu(t)\left(1+\frac{1}{\Gamma(2-\delta)}\right) \nu}{\Gamma(\gamma+1)}\right]+\frac{\left(l v-F_{0}\right)(c)}{\Gamma(\gamma+1)}\left(1+\frac{1}{\Gamma(2-\delta)}\right)+\sum_{i=1}^{m} \frac{n_{i}}{\Gamma\left(\zeta_{i}+1\right)}\right] } \\
& \times\left\|\phi_{1}-\phi_{2}\right\| .
\end{aligned}
$$

Proof Let $x$ and $y$ be two solutions of equation (1). Then from equation (2) we have

$$
\begin{aligned}
|x(t)-y(t)| \leq & \mid f\left(t, x_{t}\right)\left[N+\frac{1}{\Gamma(\gamma)} \int_{0}^{t}(t-s)^{\gamma-1} g\left(s, x_{s},{ }^{c} D_{0^{+}}^{\delta} x_{s}\right) d s\right] \\
& -f\left(t, y_{t}\right)\left[N+\frac{1}{\Gamma(\gamma)} \int_{0}^{t}(t-s)^{\gamma-1} g\left(s, y_{s},{ }^{c} D_{0^{+}}^{\delta} y_{s}\right) d s\right] \\
& +\left[\sum_{i=1}^{m} I^{\zeta i} h_{i}\left(t, x_{t}\right)-\sum_{i=1}^{m} I^{\zeta i} h_{i}\left(t, y_{t}\right)\right] \mid \\
\leq & \left|f\left(t, x_{t}\right)-f\left(t, y_{t}\right)\right|\left[\left|N+\frac{1}{\Gamma(\gamma)} \int_{0}^{t}(t-s)^{\gamma-1} g\left(s, x_{s},{ }^{c} D_{0^{+}}^{\delta} x_{s}\right) d s\right|\right] \\
& +f\left(t, y_{t}\right)\left[\frac{1}{\Gamma(\gamma)} \int_{0}^{t}(t-s)^{\gamma-1}\left|g\left(s, x_{s},{ }^{c} D_{0^{+}}^{\delta} x_{s}\right) d s-g\left(s, y_{s}{ }^{c} D_{0^{+}}^{\delta} y_{s}\right)\right|\right] \\
& +\sum_{i=1}^{m} \frac{n_{i}}{\Gamma\left(\zeta_{i}+1\right)}\left\|\phi_{1}-\phi_{2}\right\| .
\end{aligned}
$$

Now consider

$$
\begin{aligned}
I^{\gamma}\left|g\left(t, x_{t},{ }^{c} D_{0^{+}}^{\delta} x_{t}\right)-g\left(t, y_{t},{ }^{c} D_{0^{+}}^{\delta} y_{t}\right)\right| \leq & I^{\gamma} c\left[\left|x_{t}-y_{t}\right|+\left|{ }^{c} D_{0^{+}}^{\delta} x_{t}-{ }^{c} D_{0^{+}}^{\delta} y_{t}\right|\right] \\
\leq & c \frac{1}{\Gamma(\gamma)} \int_{0}^{t}(t-s)^{\gamma-1}\left|x_{s}-y_{s}\right| d s \\
& +c \frac{1}{\Gamma(\gamma)} \int_{0}^{t}(t-s)^{\gamma-1}\left|{ }^{c} D_{0^{+}}^{\delta} x_{s}-{ }^{c} D_{0^{+}}^{\delta} y_{s}\right| d s \\
\leq & \frac{c}{\Gamma(\gamma+1)}\left\|x_{t}-y_{t}\right\|+\frac{c}{\Gamma(\gamma+1) \Gamma(2-\delta)}\left\|x_{t}-y_{t}\right\| \\
\leq & \left(\frac{c}{\Gamma(\gamma+1)}\right)\left(1+\frac{1}{\Gamma(2-\delta)}\right)\left\|\phi_{1}-\phi_{2}\right\| .
\end{aligned}
$$

So equation (8) implies that

$$
\begin{aligned}
|x(t)-y(t)| \leq & l\left\|x_{t}-y_{t}\right\|\left[N+\frac{1}{\Gamma(\gamma)} \mu(t)\left(\left\|x_{t}\right\|+\left\|{ }^{c} D_{0^{+}}^{\delta} x_{t}\right\|\right) \int_{0}^{t}(t-s)^{\gamma-1} d s\right] \\
& +\left[\left|f\left(t, y_{t}\right)-f(t, 0)\right|+|f(t, 0)|\right]\left[\left(\frac{c}{\Gamma(\gamma+1)}\right)\left(1+\frac{1}{\Gamma(2-\delta)}\right)\left\|\phi_{1}-\phi_{2}\right\|\right] \\
& +\sum_{i=1}^{m} \frac{n_{i}}{\Gamma\left(\zeta_{i}+1\right)}\left\|\phi_{1}-\phi_{2}\right\| \\
\leq & l\left[N+\frac{\mu(t)\left(1+\frac{1}{\Gamma(2-\delta)}\right) v}{\Gamma(\gamma+1)}\right]\left\|\phi_{1}-\phi_{2}\right\|+\left[l\left\|y_{t}\right\|+F_{0}\right]
\end{aligned}
$$




$$
\begin{aligned}
& \times\left[\frac{c}{\Gamma(\gamma+1)}\left(1+\frac{1}{\Gamma(2-\delta)}\right)\right]\left\|\phi_{1}-\phi_{2}\right\|+\sum_{i=1}^{m} \frac{n_{i}}{\Gamma\left(\zeta_{i}+1\right)}\left\|\phi_{1}-\phi_{2}\right\| \\
\leq & {\left[l\left[N+\frac{\mu(t)\left(1+\frac{1}{\Gamma(2-\delta)}\right) v}{\Gamma(\gamma+1)}\right]+\frac{\left(l v-F_{0}\right)(c)}{\Gamma(\gamma+1)}\left(1+\frac{1}{\Gamma(2-\delta)}\right)\right.} \\
& \left.+\sum_{i=1}^{m} \frac{n_{i}}{\Gamma\left(\zeta_{i}+1\right)}\right]\left\|\phi_{1}-\phi_{2}\right\| .
\end{aligned}
$$

Corollary 1 Under the assumptions of Theorem 4.1, the solution of equation (1) is unique.

Proof Let $x$ and $y$ be two solutions of equation (1). Then from Theorem 4.1 we have that $\|x-y\|=0$ (since $\phi_{1}=\phi_{2}=\phi$ in this case). Hence the uniqueness.

\section{Example}

In this section, we present an example to explain the applicability of the main results.

Example 1 Consider the following initial value problem of fractional neutral hybrid integro-differential equation:

$$
\begin{cases}{ }^{c} D_{0^{+}}^{\frac{3}{4}}\left[\frac{x(t)-\sum_{i=1}^{4} \frac{2 i-1}{2} h_{i}\left(t, x_{t}\right)}{1+\frac{\sinh }{12}|x t|}\right]=\left(1+\left|x_{t}\right|+{ }^{c} D_{0^{+}}^{\frac{1}{2}}\left|x_{t}\right|\right), & t \in[0,1], \\ x(t)=1, & -\pi \leq t \leq 0\end{cases}
$$

with

$$
h_{i}\left(t, x_{t}\right)=\frac{\left|x_{t}\right|}{(12+2 i+t)\left(1+\left|x_{t}\right|\right)}
$$

and $\zeta_{i}=\frac{2 i-1}{2}, i=1,2,3,4$. Therefore $\zeta_{1}=\frac{1}{2}, \zeta_{2}=\frac{3}{2}, \zeta_{3}=\frac{5}{2}, \zeta_{4}=\frac{7}{2}, \gamma=\frac{3}{4}, \delta=\frac{1}{2}$,

$$
\begin{aligned}
\left|f\left(t, x_{t}\right)-f\left(t, y_{t}\right)\right| & =\left|\left(1+\frac{\sin t}{12}\left|x_{t}\right|\right)-\left(1+\frac{\sin t}{12}\left|y_{t}\right|\right)\right| \\
& \leq \frac{\sin t}{12}\left\|x_{t}-y_{t}\right\|,
\end{aligned}
$$

and

$$
\begin{aligned}
\left|h_{i}\left(t, x_{t}\right)-h_{i}\left(t, y_{t}\right)\right| & =\frac{1}{24+i+t}\left|\frac{\left|x_{t}\right|}{1+\left|x_{t}\right|}-\frac{\left|y_{t}\right|}{1+\left|y_{t}\right|}\right| \\
& \leq \frac{1}{24+i+t}\left\|x_{t}-y_{t}\right\| .
\end{aligned}
$$

From this we get the norms $\|l\|=\frac{1}{12}$ and $\left\|n_{i}\right\|=\frac{1}{13+2 i}$. Moreover,

$$
\begin{aligned}
h_{i}(0, \phi(\theta)) & =\frac{|\phi(\theta)|}{(12+2 i)(1+|\phi(\theta)|)} \\
& =\frac{1}{(12+2 i)(2)}, \quad i=1,2,3,4,
\end{aligned}
$$


and therefore $N=1-I^{\frac{1}{2}}\left(\frac{1}{28}\right)-I^{\frac{3}{2}}\left(\frac{1}{32}\right)-I^{\frac{5}{2}}\left(\frac{1}{36}\right)-I^{\frac{7}{2}}\left(\frac{1}{40}\right)$. By using $I^{\zeta} c=\frac{t^{\zeta}}{\Gamma(\zeta+1)}$ we get $N \approx$ 0.0743, $\left(1+\frac{1}{\Gamma(2-\delta)}\right)(v)=0.4698 v, F_{0}=1, K_{0}=0, \mu(s)=1$. Also, $\sum_{i=1}^{4} \frac{n_{i}}{\Gamma\left(\zeta_{i}+1\right)}=0.1393$. So

$$
v \geq \frac{F_{0}\left[N+\frac{\mu(s)\left(1+\frac{1}{\Gamma(2-\delta)}\right) v}{\Gamma(\gamma+1)}\right]+\sum_{i=1}^{m} \frac{K_{0}}{\Gamma\left(\zeta_{i}+1\right)}}{1-N l-l \frac{\mu(s)\left(1+\frac{1}{\Gamma(2-\delta)}\right) v}{\Gamma(\gamma+1)}-\sum_{i=1}^{m} \frac{n_{i}}{\Gamma\left(\zeta_{i}+1\right)}}
$$

implies that $v \in[0.2242,7.8345]$. Hence all the conditions of Theorem 3.1 are satisfied. Therefore problem (9) has at least one solution on $[0,1]$.

\section{Conclusions}

In this paper, we proved the existence of a solution for initial value problem for a class of hybrid fractional differential equation with delay by using a hybrid fixed point theorem of Dhage [24] for three operators in a Banach algebra. We also derived the data dependence of a solution. The main result is well illustrated by an example.

\section{Competing interests}

The authors declare to have no competing interests.

\section{Authors' contributions}

The authors read and approved the final manuscript.

\section{Authors' information}

This research had been supported by National Natural Science Foundation of China (nos. 11371027, 11471015, and 11601003)

\section{Author details}

${ }^{1}$ School of Mathematical Sciences, Anhui University, Hefei, Anhui 230039, China. ${ }^{2}$ Department of Mathematics, School of Natural Sciences (SNS), National University of Sciences and Technology (NUST), H-12 Sector, Islamabad, 44000, Pakistan.

\section{Publisher's Note}

Springer Nature remains neutral with regard to jurisdictional claims in published maps and institutional affiliations.

Received: 1 July 2017 Accepted: 19 October 2017 Published online: 02 November 2017

\section{References}

1. Anguraj, A, Karthikeyan, P, Rivero, M, Trujillo, JJ: On new existence results for fractional integro-differential equations with impulsive and integral conditions. Comput. Math. Appl. 66(12), 2587-2594 (2014)

2. Arshad, S, Lupulescu, V, Regan, DÓ: $L_{p}$-Solutions for fractional integral equations. Fract. Calc. Appl. Anal. 17(1), $259-276$ (2014)

3. Harikrishnan, S, Prakash, P, Nieto, JJ: Forced oscillation of solutions of a nonlinear fractional partial differential equation. Appl. Math. Comput. 254, 14-19 (2015)

4. Li, K, Peng, J, Gao, J: Nonlocal fractional semilinear differential equations in separable Banach spaces. Electron. J. Differ. Equ. 2013, 7 (2013).

5. Rodríguez-López, R, Tersian, S: Multiple solutions to boundary value problem for impulsive fractional differential equations. Fract. Calc. Appl. Anal. 17(4), 1016-1038 (2014)

6. Wang, JR, Zhou, Y, Fečkan, M: Abstract Cauchy problem for fractional differential equations. Nonlinear Dyn. 71, 685-700 (2013)

7. Zhang, Z, Ning, Q, Wang, H: Mild solutions of fractional evolution equations on an unbounded interval. Adv. Differ. Equ. 2014, 27 (2014)

8. Sheng, J, Jiang, W: Existence and uniqueness of the solution of fractional damped dynamical systems. Adv. Differ. Equ. 2017, 16 (2017)

9. Ahmad, B, Ntouyas, SK: Nonlocal fractional boundary value problems with slit-strips boundary conditions. Fract. Calc. Appl. Anal. 18, 261-280 (2015)

10. Chen, L, Wu, R, Chu, Z, He, Y: Stabilization of fractional-order coupled systems with time delay on networks. Nonlinear Dyn. 88(1), 521-528 (2017)

11. Chen, L, Wu, R, Cao, J, Liu, JB: Stability and synchronization of memristor-based fractional-order delayed neural networks. Neural Netw. 71, 37-44 (2015)

12. Chen, L, Liu, C, Wu, R, He, Y, Chai, Y: Finite-time stability criteria for a class of fractional-order neural networks with delay. Neural Comput. Appl. 27(3), 549-556 (2016)

13. Kilbas, AA, Srivastava, HM, Trujillo, JJ: Theory and Applications of Fractional Differential Equations. North-Holland Mathematics Studies, vol. 204. Elsevier Science BV, Amsterdam (2006) 
14. Podlubny, I: Fractional Differential Equations. Academic Press, San Diego (1999)

15. Sabatier, J, Agrawal, RP, Machado, JAT (eds.): Advances in Fractional Calculus: Theoretical Developments and Applications in Physics and Engineering. Springer, Dordrecht (2007)

16. Lakshmikantham, V, Leela, S: Differential and Integral Inequalities. Academic Press, New York (1969)

17. Dhage, BC: On $\alpha$-condensing mappings in Banach algebras. Math. Stud. 63, 146-152 (1994)

18. Dhage, BC, Lakshmikantham, V: Basic results on hybrid differential equations. Nonlinear Anal., Real World Appl. 4 414-424 (2010)

19. Dhage, BC: A nonlinear alternative in Banach algebras with applications to functional differential equations. Nonlinear Funct. Anal. Appl. 8, 563-575 (2004)

20. Dhage, BC: Fixed point theorems in ordered Banach algebras and applications. Panam. Math. J. 9, 93-102 (1999)

21. Dhage, BC: Quadratic perturbations of periodic boundary value problems of second order ordinary differential equations. Differ. Equ. Appl. 2, 465-486 (2010)

22. Dhage, BC: Basic results in the theory of hybrid differential equations with mixed perturbations of second type. Funct. Differ. Equ. 19, 1-20 (2012)

23. Dhage, BC, Ntouyas, SK: Existence results for boundary value problems for fractional hybrid differential inclusions. Topol. Methods Nonlinear Anal. 44, 229-238 (2014)

24. Dhage, BC: A fixed point theorem in Banach algebras with applications to functional integral equations. Kyungpook Math. J. 44, 145-155 (2004)

25. Lu, H, Sun, S, Yang, D, Teng, H: Theory of fractional hybrid differential equations with linear perturbations of second type. Boundary Value Problems, 23 (2013)

26. Zhao, Y, Sun, S, Han, Z, Li, Q: Theory of fractional hybrid differential equations. Comput. Math. Appl. 62, 1312-1324 (2011)

27. Sitho, S, Ntouyas, SK, Tariboon, J: Existence results for hybrid fractional integro-differential equations. Boundary Value Problems, 113 (2015)

28. Niazi, AUK, Jiang, W, Rehman, M, Pang, D: Boundary value problem for nonlinear fractional differential equations with delay. Advances in Difference Equations, 24 (2017)

29. Sun, S, Zhao, Y, Han, Z, Li, Y: The existence of solutions for boundary value problem of fractional hybrid differential equations. Commun. Nonlinear Sci. Numer. Simul. 17, 4961-4967 (2012)

30. Ahmad, B, Ntouyas, SK: An existence theorem for fractional hybrid differential inclusions of Hadamard type with Dirichlet boundary conditions. Abstr. Appl. Anal. 2014, Article ID 705809 (2014)

31. Ahmad, B, Ntouyas, SK, Alsaedi, A: Existence results for a system of coupled hybrid fractional differential equations. Sci. World J. 2014, Article ID 426438 (2014)

32. Ahmad, B, Ntouyas, SK, Tariboon, J: A nonlocal hybrid boundary value problem of Caputo fractional integro-differential equations. Acta Math. Sci. 36B(6), 1631-1640 (2016)

33. Abbas, S: Existence of solutions to fractional order ordinary and delay differential equations and applications. Electron. J. Differ. Equ. 2011, 9 (2011)

\section{Submit your manuscript to a SpringerOpen ${ }^{\circ}$ journal and benefit from:}

- Convenient online submission

- Rigorous peer review

- Open access: articles freely available online

- High visibility within the field

- Retaining the copyright to your article

Submit your next manuscript at $>$ springeropen.com 